
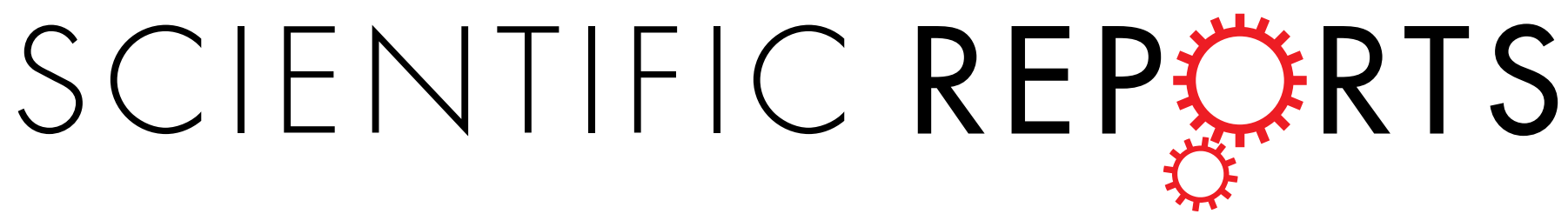

\title{
OPEN Asymmetric Waveforms Decrease Lethal Thresholds in High Frequency Irreversible \\ Electroporation Therapies
}

Received: 05 May 2016

Accepted: 12 December 2016

Published: 20 January 2017

\author{
Michael B. Sano ${ }^{1,2}$, Richard E. Fan ${ }^{3}$ \& Lei Xing ${ }^{1}$
}

Irreversible electroporation (IRE) is a promising non-thermal treatment for inoperable tumors which uses short (50-100 $\mathrm{s}$ ) high voltage monopolar pulses to disrupt the membranes of cells within a welldefined volume. Challenges with IRE include complex treatment planning and the induction of intense muscle contractions. High frequency IRE (H-FIRE) uses bursts of ultrashort $(0.25-5 \mu \mathrm{s})$ alternating polarity pulses to produce more predictable ablations and alleviate muscle contractions associated with IRE. However, H-FIRE generally ablates smaller volumes of tissue than IRE. This study shows that asymmetric $H$-FIRE waveforms can be used to create ablation volumes equivalent to standard IRE treatments. Lethal thresholds (LT) of $505 \mathrm{~V} / \mathrm{cm}$ and $1316 \mathrm{~V} / \mathrm{cm}$ were found for brain cancer cells when $100 \mu s$ IRE and $2 \mu s$ symmetric H-FIRE waveforms were used. In contrast, LT as low as $536 \mathrm{~V} / \mathrm{cm}$ were found for $2 \mu$ s asymmetric H-FIRE waveforms. Reversible electroporation thresholds were $54 \%$ lower than LTs for symmetric waveforms and 33\% lower for asymmetric waveforms indicating that waveform symmetry can be used to tune the relative sizes of reversible and irreversible ablation zones. Numerical simulations predicted that asymmetric $\mathrm{H}$-FIRE waveforms are capable of producing ablation volumes which were 5.8-6.3x larger than symmetric H-FIRE waveforms indicating that in vivo investigation of asymmetric waveforms is warranted.

Irreversible electroporation (IRE) is an emerging cancer therapy which uses high intensity electrical pulses to focally ablate solid tumors ${ }^{1}$. Clinically, two or more needle electrodes are advanced around a target tumor. A series of approximately 100 electrical pulses, 1000 to $3000 \mathrm{~V}$ in amplitude and 50 to $100 \mu$ s in duration, are then delivered. These electrical pulses locally increase cell transmembrane potentials above a critical lethal threshold to create permanent nanoscale defects, which result in rapid cell death. IRE ablations exhibit a characteristic sub-millimeter transition between complete cell death to unaffected tissue due to the rapid change in electric field intensity near the electrodes.

Small cohort studies of IRE for the treatment of inoperable tumors smaller than $3 \mathrm{~cm}$ show complete response rates between 93 and $98 \%$. These favorable early clinical results have encouraged significant interest in the use of IRE for the treatment of liver ${ }^{4}$, pancreatic ${ }^{5-7}$, kidney $^{8}$, prostate ${ }^{9-13}$, and brain ${ }^{14-17}$ tumors.

Though IRE is a promising emerging procedure, there are some clinical challenges which may impede widespread adoption of the therapy. The long duration electrical pulses create local and systemic muscle contractions and may inadvertently interact with cardiac rhythms ${ }^{18}$. To alleviate this, patients must receive significant doses of chemical paralytics and pulse delivery is synchronized with the heart-beat to ensure pulses are delivered during the absolute refractory period ${ }^{19}$. This is challenging since it significantly changes the clinical workflow in comparison to other focal therapies and can increase the overall treatment time. Additionally, the ablation zone produced by IRE is dependent on local dynamic electrical properties of the tissue and heterogeneities can potentially distort the electric field and produce irregular shaped ablations ${ }^{20}$.

High frequency irreversible electroporation (H-FIRE) is a new protocol designed to address these challenges. H-FIRE replaces the long duration monopolar IRE pulses (Fig. 1a) with a burst of alternating polarity pulses

${ }^{1}$ Stanford University Medical Center, Department of Radiation Oncology, Division of Radiation Physics, Stanford, CA, USA. ${ }^{2}$ UNC / NCSU Joint Department of Biomedical Engineering, Chapel Hill, NC, USA. ${ }^{3}$ Stanford University Medical Center, Department of Urology, Stanford, CA, USA. Correspondence and requests for materials should be addressed to M.B.S. (email: mikesano@stanford.edu) 

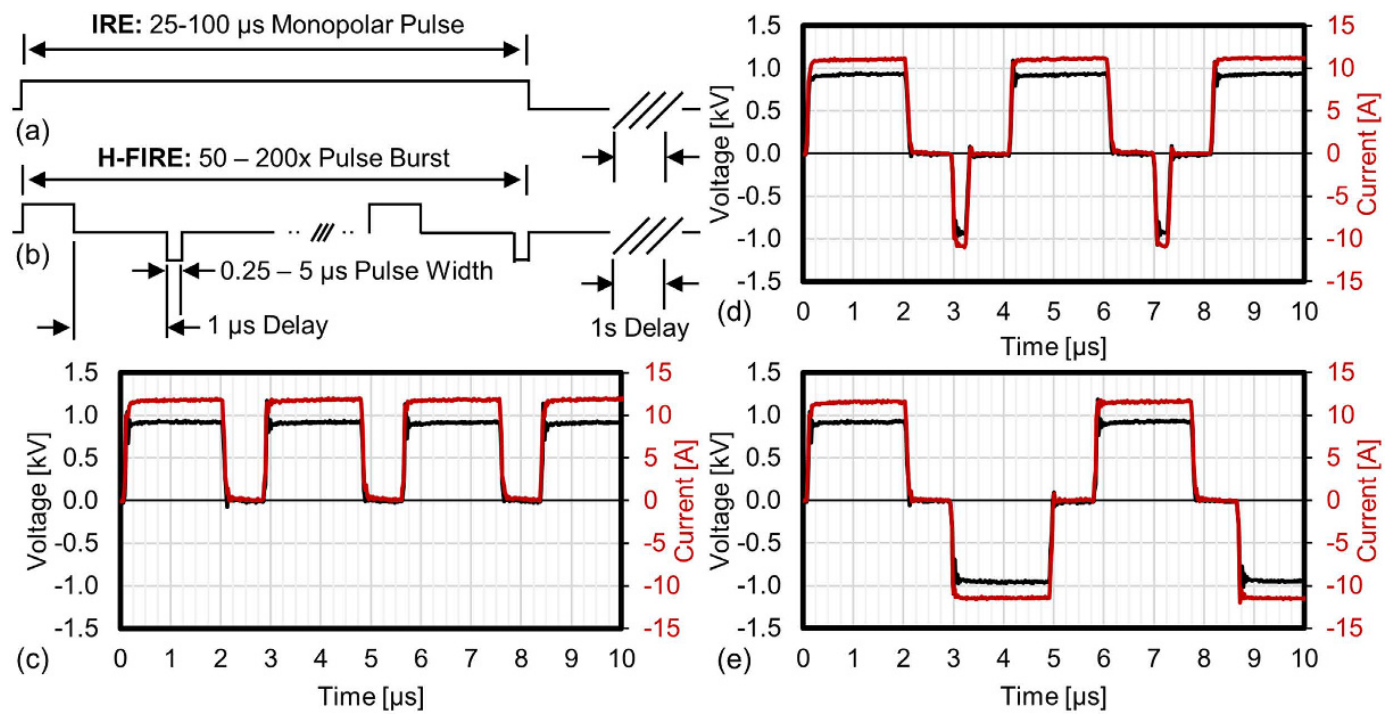

Figure 1. IRE and H-FIRE Use Different Pulse Waveforms: (a) IRE treatments deliver a series of long duration monopolar pulses. (b) H-FIRE treatments typically deliver multiple bursts containing 0.25 to $5 \mu$ s alternating polarity pulses. In this study we examine the effects of pulse symmetry. Example waveforms showing the first $10 \mu \mathrm{s}$ of a (c) $2 \mu \mathrm{s}$ monopolar, (d) asymmetric $2-1-0.25 \mu \mathrm{s}$ (positive - delay - negative), and (e) symmetric 2-1-2 (positive - delay - negative) H-FIRE bursts. These waveforms are significantly more complex than standard IRE pulses and variations in the configuration of H-FIRE bursts impact treatment outcomes.

between $250 \mathrm{~ns}$ and $5 \mu \mathrm{s}$ (Fig. 1b). The electrical conductivities of skin, dura, tendons, blood vessels, and glandular tissues appear to converge at frequencies around $1 \mathrm{MHz}^{21}$ resulting in H-FIRE ablations which more closely match analytical predictions in simple tissue model ${ }^{22}$. Numerical analysis predict that these waveforms can short through skin and fat layers to produce more uniform ablations than IRE ${ }^{23}$. H-FIRE waveforms effectively eliminate muscle contractions in small animal model ${ }^{24}$ and these treatments have been used to treat spontaneous tumors in large animals without the need for general anesthesia or chemical paralysis ${ }^{25}$. H-FIRE treatments have been shown to significantly inhibit tumor growth in murine flank tumor models ${ }^{25}$ and it was recently demonstrated that H-FIRE treatments preferentially target malignant cells in co-cultured 3D tumor models ${ }^{26}$. Limitations of H-FIRE include the ablation size, which is typically smaller than with conventional IRE and the need for specialized electronics which can deliver the appropriate alternating polarity electrical pulses.

In vivo, IRE is typically observed in regions which are exposed to approximately $500-750 \mathrm{~V} / \mathrm{cm}^{14,27,28}$. Preliminary studies indicate that for equivalent energy treatments the lethal electric field is higher for H-FIRE than for IRE treatments ${ }^{25,26,29}$. Using a 3D in vitro pancreatic tumor model, Arena et al. found a lethal threshold of $500 \mathrm{~V} / \mathrm{cm}$ when $80 \times 100 \mu \mathrm{s}$ IRE pulses were delivered ${ }^{30}$. The lethal electric field thresholds for equivalent energy $\mathrm{H}$-FIRE protocols in the same model were much higher, at 2022,1687, 1070, and $755 \mathrm{~V} / \mathrm{cm}$ for bursts containing $0.25,0.5,1$, and $2 \mu$ s symmetric waveforms, respectively ${ }^{25}$. Given this increase in lethal threshold, to successfully treat $1-3 \mathrm{~cm}$ tumors, these H-FIRE protocols would require a pulse generator which outputs significantly higher voltages than the $3000 \mathrm{~V}$ maximum currently implemented in the clinic.

Given these practical limitations, we present an alternate approach to decrease lethal thresholds and increase ablation volumes through the use of asymmetric pulse waveforms. Three experiments were conducted to evaluate the implications of pulse asymmetry in electroporation therapies. First, to investigate the impacts of culture conditions on IRE and H-FIRE outcomes, U87 cells were grown in either 2D monolayer or 3D cultures and exposed to an equivalent delivered energy in several different waveforms. These preliminary experiments mirror clinical protocols which deliver $100 \mu$ s monopolar pulses 100 times. Second, to demonstrate clinical utility against multiple forms of cancer, the lethal thresholds for U87 and MDA-MB-231 BR3 cells were examined in response to a broad array of pulse waveforms, comparing duration of energized bursts and pulse asymmetry. Third, to investigate if waveform asymmetry has any relevant implications for electro-chemotherapy (ECT) $)^{31}$ or electro-gene therapy $(\mathrm{EGT})^{32}$, reversible electroporation thresholds were evaluated by adding membrane impermeable propidium iodide (PI) to 2D monolayers of each cell type prior to treatment. In this third experiment, cell cultures were exposed to lower energy treatments with 100x bursts where each burst was energized for $50 \mu \mathrm{s}$. Finally, the reversible and lethal thresholds found in vitro were incorporated into a three-dimensional finite element model to predict the size and shape of ablations which would be created by H-FIRE waveforms if $3 \mathrm{kV}$ pulses were delivered into live tissue through clinical electrodes. These models were then validated against ablations created in ex vivo liver tissue.

It was found that both $2 \mathrm{D}$ and $3 \mathrm{D}$ culture conditions adequately replicate IRE lethal thresholds found in vivo and that $2 \mathrm{D}$ cultures are acceptable for investigating H-FIRE protocols. Waveforms which incorporate asymmetry had lower lethal thresholds and result in larger ablation zones than symmetric H-FIRE waveforms (Fig. 1c-e). This indicates that the elevated lethal thresholds previously found in H-FIRE treatments are likely the result of waveform symmetry, and reduced lethal thresholds can be achieved with asymmetric H-FIRE waveforms. It was 

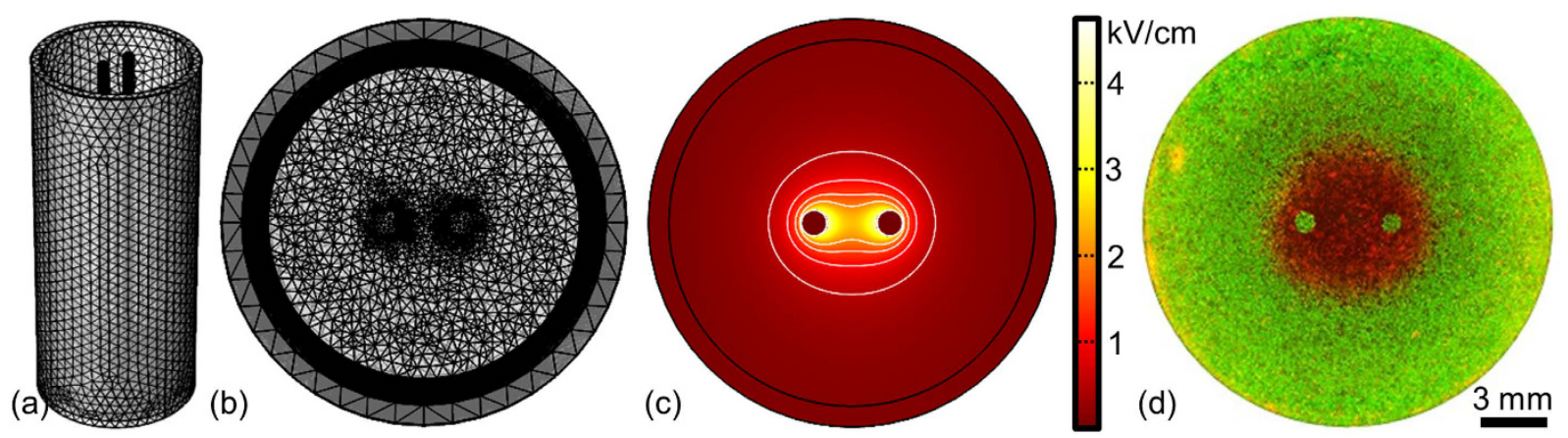

Figure 2. Pulsed Electric Fields Affect Cells within a Well Defined Treatment Zone which is Dependent on the Electrode Configuration and Pulse Parameters. (a,b) Mesh used to simulate the electric field distribution within a 12 -well when $1 \mathrm{~mm}$ diameter electrodes spaced $3 \mathrm{~mm}$ edge-to-edge are used to deliver $900 \mathrm{~V}$. (c) Electric field distribution within the well. Isocontour lines represent electric fields of 500, 1000, 1500, and $2000 \mathrm{~V} / \mathrm{cm}$ [outside to inside]. (d) Electroporated [Red] and unaffected [Green] MDA-MB-231 BR3 cells immediately post-treatment after exposure to 100x H-FIRE Bursts. Each burst used a 2-1-0.5 $\mu$ s waveform (positive - delay - negative time) which was energized for $50 \mu \mathrm{s}$. These numerical simulations were used to determine the electric field required to produce the focal treatment zone created in vitro.

found that the differences between reversible electroporation and lethal thresholds were substantially larger for symmetric waveforms than for asymmetric waveforms indicating that ECT and EGT treatment protocols may benefit from the use of symmetric waveforms. Finally, numerical models using experimentally determined lethal thresholds indicate that asymmetric H-FIRE waveforms can be used to produce 5.8-6.3x larger ablation volumes than symmetric waveforms. These results indicate that waveform asymmetry is a useful new parameter which can be used to modulate treatment outcomes and in vivo investigation of these waveforms is warranted.

\section{Methods}

Pulse Delivery. All treatments were delivered through two blunt $1.29 \mathrm{~mm}$ diameter (16 gauge) stainless steel needles with a $3 \mathrm{~mm}$ edge-to-edge separation. A custom pulse generator was used to deliver IRE and H-FIRE protocols which were either monopolar: all pulses were positive polarity (Fig. 1c), asymmetric bipolar: positive and negative pulses had different durations (Fig. 1d), or symmetric bipolar: positive and negative pulses were the same duration (Fig. 1e). To simplify discussion of these protocols we use the notation $\mathrm{P}-1-\mathrm{N}$ where $\mathrm{P}$ indicates the positive pulse length and $\mathrm{N}$ indicates the negative pulse length in microseconds. All protocols used a $1 \mu \mathrm{s}$ delay between changes in pulse polarity.

For example, $2-1-0.25$ represents a pulse waveform with a $2 \mu$ s positive pulse, followed by a $1 \mu$ s delay, then a $0.25 \mu$ s negative pulse as shown in Fig. $1 \mathrm{~d}$. To compare equivalent energy IRE and H-FIRE treatments, the H-FIRE waveforms were repeated in a burst such that the total energized time of each burst was equivalent to 50 or $100 \mu \mathrm{s}$. In cases where waveforms could not produce energized times of exactly 50 or $100 \mu$ s, the closest energized time without exceeding the target time was used. In all treatment groups bursts were delivered with a repetition rate of $1 \mathrm{~Hz}$ and the output of the generator was set to deliver pulses which were $900 \mathrm{~V}$ in amplitude.

Cell Culture. In vitro experiments were conducted on a glioblastoma cell line (U87, American Type Culture Collection Inc. Manassas, VA) and cells isolated from murine brain metastases after inoculation with human breast cancer cells (MDA-MB-231 BR3, a generous gift from the Laboratory of Martin Brown, Stanford, CA) ${ }^{33}$. These cells were chosen to model primary and metastatic brain tumors, respectively, and to evaluate potential changes in treatment response by cells of different disease origins. The cells were cultured to $80 \%$ confluency in High Glucose Dulbecco's Modified Eagle Medium with L-Glutamine (catalog number 12800-082, Invitrogen Inc., Carlsbad, CA), which was supplemented with 10\% heat inactivated Fetal Calf Serum (catalog number S11150, lot G14092, Atlanta Biologicals Inc., Lawrenceville, GA), 0.2 g/L Streptomycin Sulfate (catalog number 11860038, Invitrogen Inc., Carlsbad, CA), and 0.126 g/L Penicillin G Potassium Salt (catalog number P7794-100MU, Sigma-Aldrich Inc., St. Louis, MO). Cells were harvested via trypsinization and seeded either directly onto the bottom of 12 well plates (2D culture) or in a 50\% mixture of Matrigel (catalog number 356235, Corning Inc., Corning, NY) and culture media (3D culture) at a density of $100 \mathrm{k}$ cells $/ \mathrm{mL}$. These cells were then allowed to reach confluent cultures over 4-7 days over which time cell culture media was replaced as necessary. Prior to treatment, media was removed and replaced with $2 \mathrm{~mL}$ of fresh culture media.

Assessment of Reversible and Irreversible Electroporation. Stock solutions of $4 \mu \mathrm{M}$ Calcein AM (Life Technologies, Carlsbad, CA) were prepared by adding $125 \mu \mathrm{L}$ of sterile dimethyl sulfoxide to $50 \mu \mathrm{g}$ of powdered dye and stored at $-20^{\circ} \mathrm{C}$ until use. Stock solutions of propidium iodide (PI) (MP Biomedicals Inc., Burlingame, CA) were prepared using a concentration of $1 \mathrm{mg} / \mathrm{mL}$ PI in sterile phosphate-buffered saline and stored at $4{ }^{\circ} \mathrm{C}$ until use. Reversible electroporation thresholds were assessed by adding $100 \mu \mathrm{L} / \mathrm{mL}$ of PI stock solutions to each well 5 minutes prior to treatment. The samples were imaged immediately after treatment using a fluorescence microscope with an automated stage (Leica DMI600 B, Buffalo Grove, IL) using a red fluorescent protein filter cube $(540-552 \mathrm{~nm} / 580-620 \mathrm{~nm})$ to visualize the electroporated population which had been 
(a)

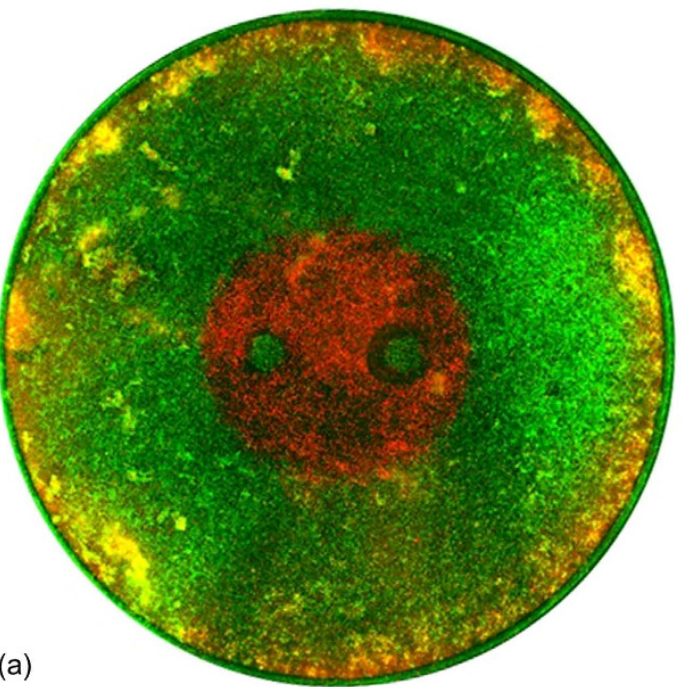

(b)

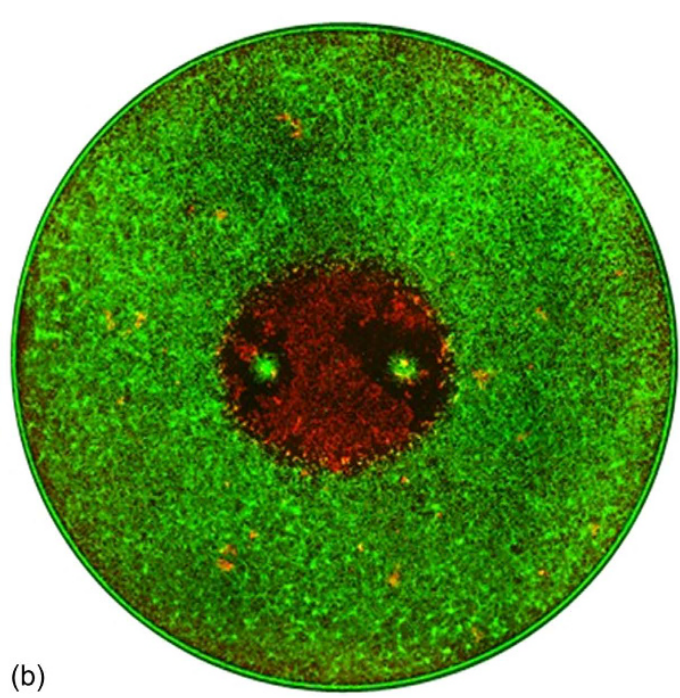

Figure 3. Comparison of ablations in 3D and 2D culture conditions. U87 cells were grown in (a) 3D Matrigel or (a) 2D media-only culture conditions. After reaching confluency cells were exposed to H-FIRE protocols. Live (green) and dead (red) images were obtained 24 hours post treatment to determine the ablation geometry. For most treatment groups, there was no statistical difference in the lethal threshold between $2 \mathrm{D}$ and $3 \mathrm{D}$ culture. Regions of live and dead cells are visible around the perimeter of the 3D cultures where the Matrigel forms a thick meniscus which may result in hypoxic conditions ${ }^{30}$. This comparative study indicates that $3 \mathrm{D}$ culture conditions may not be necessary to evaluate the relative effects of electroporation parameters in vitro.

counter-stained red by the PI (Fig. 2d). The height (y-axis) and width (x-axis) of each treatment zone was measured using the microscope software's built in measurement tool. To assess lethal thresholds, cells were treated with IRE or H-FIRE protocols and incubated at $37^{\circ} \mathrm{C}$ in $5 \% \mathrm{CO}_{2}$ for 24 hours prior to staining. Calcein $\mathrm{AM}(2 \mu \mathrm{L} / \mathrm{mL})$ and PI $(100 \mu \mathrm{L} / \mathrm{mL})$ stock solutions were then added to the cells and the ablation zone was imaged using green fluorescent protein $(460-500 \mathrm{~nm} / 512-542 \mathrm{~nm}$ ) and red fluorescent protein filter cubes (Fig. 3) and measured using the microscope software's built in measurement tool.

Numerical Calculation of Reversible and lethal thresholds. To calculate the reversible electroporation or lethal threshold for each experimental treatment, the electric field distribution within a standard 12 well chamber was modeled numerically using COMSOL Multiphysics (V5.2a, COMSOL Inc., Palo Alto, CA) via the 3D Electric Currents module. This module solves the equations: $\nabla \cdot \boldsymbol{J}=Q_{j}, \boldsymbol{J}=\sigma \boldsymbol{E}+\boldsymbol{J}_{e}$, and $\boldsymbol{E}=-\nabla V$ where $\mathbf{J}$ is the local current density, $\mathbf{Q}$ is the electric charge, $\mathbf{E}$ is the electric field, $\mathbf{J}_{\mathbf{e}}$ is the external current density, and $\mathrm{V}$ is the local voltage. A free tetrahedral mesh was generated in the media domain using the Extremely Fine preset while all other domains were meshed using the Normal preset values. The media domain was then refined twice using a refinement method which splits each tetrahedron along the longest edge (Fig. 2a,b). Experimental voltages were applied to the top most surface of one electrode. The top surface of the adjacent electrode was set to ground $(0 \mathrm{~V})$. All other external boundaries were set as insulators $(\boldsymbol{n} \cdot \boldsymbol{J}=0)$. The electrical conductivity $(\sigma)$ was set to $1.2 \mathrm{~S} / \mathrm{m}$ for the media, $2.22 \times 10^{6} \mathrm{~S} / \mathrm{m}$ for the electrodes, and $1 \times 10^{-16} \mathrm{~S} / \mathrm{m}$ for the plastic well plate components. These simulations required approximately 29 minutes to solve on an Intel i7 processor with 32 GB RAM.

The electric field distributions (Fig. 2c) along the $\mathrm{x}$ - and $\mathrm{y}$-axis were exported into a spreadsheet where they were used to correlate treatment geometries (Fig. 2d) with electric field values. Each treatment zone was measured and an electric field value correlating the height and width were determined. Each experimental parameter was repeated a minimum of three times $(\mathrm{N}=3)$ yielding at least six electric field values which were averaged and reported as mean \pm standard deviation. Statistical significance between groups was determined using a two-sided Student's T-test with unequal variances. An alpha value of $0.01(\alpha=0.01), 99 \%$ confidence interval, was used to determine if thresholds between treatment groups were statistically significantly different.

Finite element models were used to predict clinical ablations created by different H-FIRE waveforms (Supplemental Figure S1). These models used a dynamic tissue conductivity function ${ }^{8,14,34}$ with tissue conductivities derived from in vivo experiments ${ }^{11}$ and transitions between these conductivities centered at the reversible electroporation thresholds for each waveform found in this study. Methods for producing these models are presented in the supplemental material.

To validate these numerical models, predicted ablations were compared to ablations created in ex vivo liver tissue using NanoKnife electrodes (AngioDynamics Inc., Latham, NY). To match the simulations, a single needle and grounding pad configuration ${ }^{35}$ was used. Ex vivo porcine livers were obtained from the Stanford University Veterinary Service Center Necropsy Laboratory in accordance with the University's guidelines and regulations. Whole organs were submerged in a water tank containing a $3 \mathrm{~g} / \mathrm{L} \mathrm{NaCl}$ solution with a conductivity of $0.6 \mathrm{~S} / \mathrm{m}$. A single electrode was inserted into the tissue and a $0.254 \mathrm{~mm}$ aluminum sheet was placed $10 \mathrm{~cm}$ from 

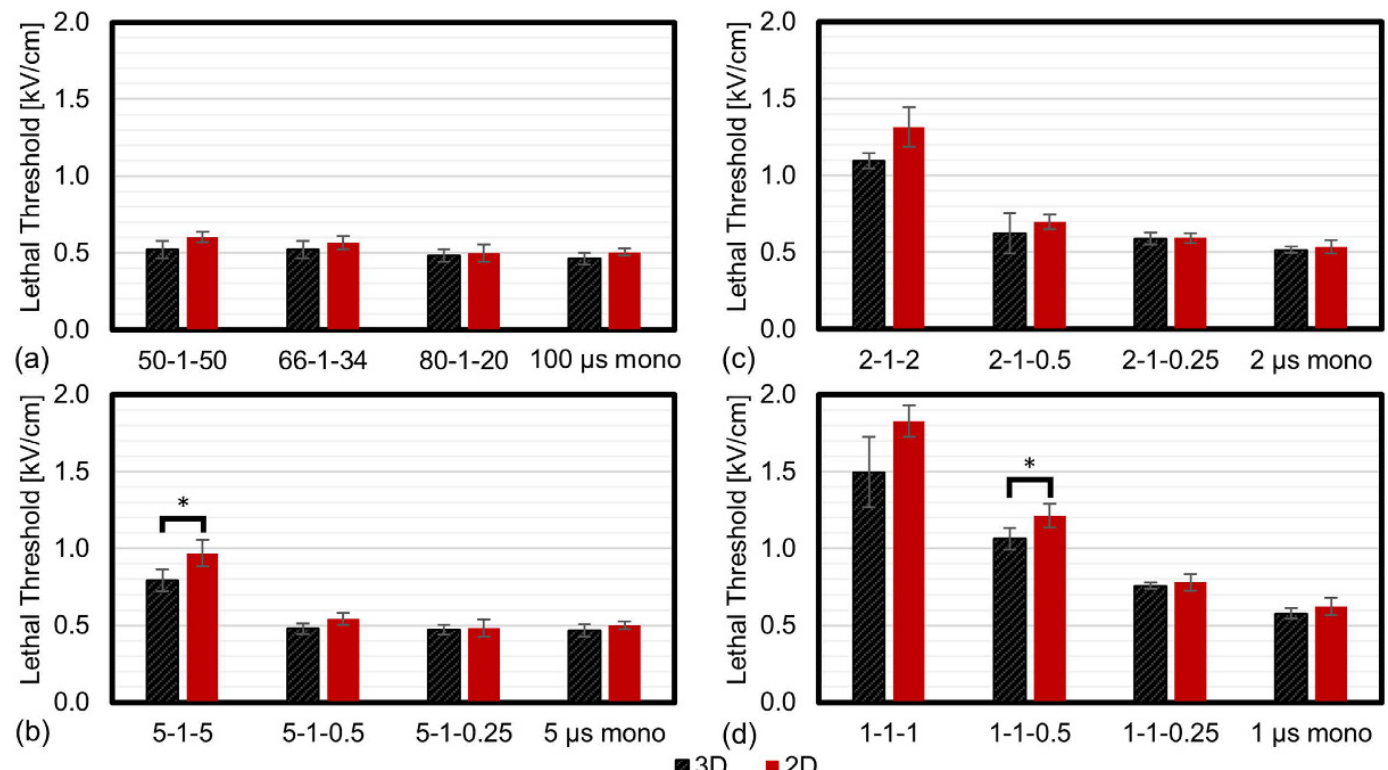

(c)

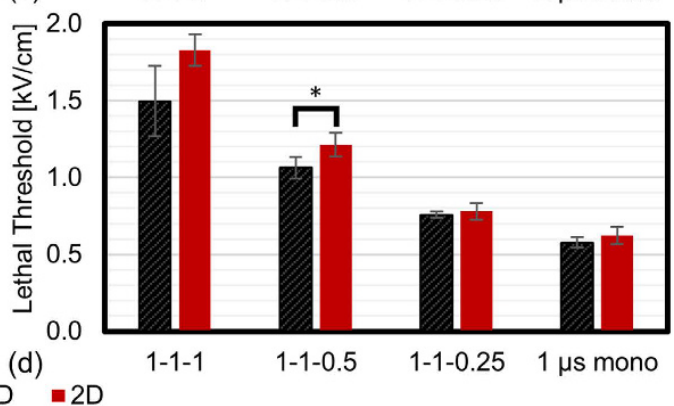

Figure 4. 2D vs 3D Culture Conditions Had Minimal Impact on Lethal Threshold for U87 cells. Examination of lethal threshold values for cells cultured in 2D and 3D environments when exposed to 100x bursts energized for $100 \mu \mathrm{s}$. (a) Lethal threshold for long duration $(>20 \mu \mathrm{s})$ IRE pulses which were either monopolar $(100 \mu \mathrm{s})$ or alternated with a $1 \mu$ s delay between polarity change. Lethal thresholds for monopolar, symmetric, and asymmetric H-FIRE pulses where the longest pulse was (b) $5 \mu \mathrm{s}$, (c) $2 \mu \mathrm{s}$, and (d) $1 \mu \mathrm{s}$. For H-FIRE treatments, symmetric waveforms had significantly higher lethal threshold than the asymmetric and monopolar waveforms. This trend existed for both $2 \mathrm{D}$ and $3 \mathrm{D}$ cultures. ${ }^{\star}$ Indicate groups which are statistically significantly different $(\alpha=0.01)$. Error bars represent one standard deviation from the mean. These results indicate that $3 \mathrm{D}$ culture conditions may not be nessary when evaluating the relative effects of electroporation pulse parameters in vitro.

the liver to serve as a current sink, simulating a distant grounding pad. $100 \times 2-1-2 \mathrm{H}$-FIRE bursts with each burst energized for $100 \mu \mathrm{s}$ at approximately $3.5 \mathrm{kV}$ were delivered to a minimum of three locations for each electrode configuration tested. The experiments were repeated using two different clinical probes for the NanoKnife, the "monopolar probe" (MP probe), a $1 \mathrm{~mm}$ diameter $1 \mathrm{~cm}$ long electrode and the "bipolar probe" (BP probe) which contains two $0.7 \mathrm{~cm}$ electrodes on a single $1.27 \mathrm{~mm}$ diameter probe. For the BP probe, the distal electrode was energized and the proximal electrode was disconnected. All experiments were conducted within four hours post-mortem. The tissue was sectioned and soaked in a $10 \mathrm{~g} / \mathrm{L}$ tetrazolium chloride (TTC) solution for 20 minutes to dye metabolically active regions red and expose regions of tissue which had been irreversibly electroporated (Supplemental Figure S2). The length and width of the ablation zones were then measured using calipers and values were reported as mean \pm standard deviation (Supplemental Figure S3).

\section{Results}

2D versus 3D Culture Conditions. A total of 16 protocols were examined in both $2 \mathrm{D}$ monolayer cultures and 3D Matrigel cultures (Fig. 4). All treatments delivered 100x bursts with each burst energized for a total of $100 \mu \mathrm{s}$. In 14 of 16 groups there was not a statistically significant difference $(\alpha=0.01)$ between the lethal thresholds found in $2 \mathrm{D}$ and $3 \mathrm{D}$ culture platforms. In the two treatments which were statistically different, the mean lethal thresholds differed by an average of $18 \%$. In groups which were not found to be statistically different, the mean lethal thresholds differed by an average of $9 \%$. For U87 cells exposed to $100 \mu$ s monopolar IRE pulses, the lethal thresholds were $464 \pm 38 \mathrm{~V} / \mathrm{cm}$ and $505 \pm 23 \mathrm{~V} / \mathrm{cm}$ for 3D and $2 \mathrm{D}$ culture, respectively. Ivey et al. found a lethal threshold of $492 \pm 41 \mathrm{~V} / \mathrm{cm}$ for U87 cells treated with an identical IRE protocol when cells were cultured in $3 \mathrm{D}$ gels made with a $2 \%$ collagen mixture ${ }^{26}$ rather than the Matrigel material used in this study. These results indicated that culture conditions had a relatively small impact on lethal thresholds in comparison to other experimental parameters (e.g. waveform symmetry). 2D culture protocols were substantially less complex and less expensive than those required for producing and maintaining 3D cultures. Based on these factors, $2 \mathrm{D}$ culture conditions were used for all additional experiments to maximize the number of experimental parameters which could be evaluated.

Effect of Waveform Asymmetry on Lethal Thresholds. The lethal thresholds for all waveforms and cell types evaluated in $2 \mathrm{D}$ culture are presented in Table 1 . Introducing asymmetry into the pulse waveform produced measurable decreases in lethal thresholds. This effect was the largest for waveforms in which the longest pulse was $1 \mu \mathrm{s}$ in duration (e.g. 1-1-1 vs. $1 \mu \mathrm{s}$ mono) and became less prominent as pulses became longer in duration. 


\begin{tabular}{|l|c|c|c|c|c|}
\hline \multirow{3}{*}{ Waveform } & U87 & MDA-MB-231 BR3 & & \multicolumn{3}{c|}{$100 \mu$ s Energized } \\
\cline { 2 - 3 } \cline { 5 - 6 } & Threshold [V/cm] & Threshold [V/cm] & Waveform & Threshold [V/cm] & Threshold [V/cm] \\
\hline $25-1-25$ & $718 \pm 61$ & $879 \pm 145$ & $50-1-50$ & $603 \pm 34$ & $725 \pm 37$ \\
\hline $33-1-17$ & $773 \pm 63$ & $876 \pm 46$ & $66-1-34$ & $567 \pm 42$ & $693 \pm 42$ \\
\hline $40-1-10$ & $684 \pm 60$ & $906 \pm 61$ & $80-1-20$ & $498^{*} \pm 57$ & $718 \pm 20$ \\
\hline $50 \mu$ s Mono & $603^{*} \pm 63$ & $779 \pm 79$ & $100 \mu s$ Mono & $505^{*} \pm 23$ & $644 \pm 55$ \\
\hline $2-1-2$ & $1702 \pm 186$ & $2028 \pm 63$ & $5-1-5$ & $967 \pm 88$ & $998 \pm 55$ \\
\hline $2-1-0.5$ & $925^{*} \pm 112$ & $1300^{*} \pm 49$ & $5-1-0.5$ & $541^{*} \pm 37$ & $826^{*} \pm 38$ \\
\hline $2-1-0.25$ & $780^{*} \pm 99$ & $971^{*} \pm 85$ & $5-1-0.25$ & $484^{*} \pm 56$ & $812^{*} \pm 72$ \\
\hline $2 \mu$ s Mono & $688^{*} \pm 58$ & $1005^{*} \pm 89$ & $5 \mu s$ Mono & $499^{*} \pm 26$ & $732^{*} \pm 82$ \\
\hline $1-1-1$ & $1839 \pm 116$ & $2761 \pm 209$ & $2-1-2$ & $1316 \pm 129$ & $1563 \pm 221$ \\
\hline $1-1-0.5$ & $1175^{*} \pm 128$ & $2782 \pm 103$ & $2-1-0.5$ & $700^{*} \pm 47$ & $885 \pm 57$ \\
\hline $1-1-0.25$ & $960^{*} \pm 114$ & $1264^{*} \pm 55$ & $2-1-0.25$ & $594^{*} \pm 32$ & $818^{*} \pm 25$ \\
\hline $1 \mu s$ Mono & $792^{*} \pm 80$ & $1051^{*} \pm 82$ & $2 \mu s$ Mono & $536^{*} \pm 43$ & $732^{*} \pm 46$ \\
\hline & & & $1-1-1$ & $1827 \pm 101$ & $2271 \pm 126$ \\
\hline & & & $1-1-0.5$ & $1213^{*} \pm 77$ & $2000 \pm 195$ \\
\hline & & & $1-1-0.25$ & $780^{*} \pm 55$ & $945^{*} \pm 59$ \\
\cline { 5 - 6 } & & & $1 \mu s$ Mono & $622^{*} \pm 56$ & $826^{*} \pm 58$ \\
\hline
\end{tabular}

Table 1. Lethal thresholds for 2D confluent monolayers of U87 and MDA-MB-231 BR3 cells exposed to 100x bursts of the listed waveforms. Each burst was energized for 50 or $100 \mu$ s and delivered at a repetition rate of $1 \mathrm{~Hz}$. Values are listed as mean \pm standard deviation. ${ }^{*}$ Indicates asymmetric waveforms which were found to be statistically significantly $(\alpha=0.01)$ different from their respective symmetric waveforms.

For U87 cells in 2D culture, the symmetric 50-1-50 and monopolar 100 $\mu$ s waveforms had lethal thresholds of $603 \pm 34 \mathrm{~V} / \mathrm{cm}$ and $505 \pm 23 \mathrm{~V} / \mathrm{cm}$, respectively (Fig. $4 \mathrm{a}$ ). A relative decrease of $98 \mathrm{~V} / \mathrm{cm}$. In contrast, the symmetric 5-1-5 and the monopolar $5 \mu$ s waveform H-FIRE waveforms had lethal thresholds of $967 \pm 88 \mathrm{~V} / \mathrm{cm}$ and $499 \pm 26 \mathrm{~V} / \mathrm{cm}$, respectively. A relative decrease of $468 \mathrm{~V} / \mathrm{cm}$. Similar results were found for the $2 \mu \mathrm{s}$ (Fig. $4 \mathrm{c}$ ) and $1 \mu \mathrm{s}$ (Fig. $4 \mathrm{~d}$ ) protocols. It is interesting to note that the monopolar $5 \mu \mathrm{s}$ and $2 \mu \mathrm{s}$ waveforms had lethal thresholds which were not significantly different $(\alpha=0.01)$ than the monopolar $100 \mu$ s waveform while the $5 \mu \mathrm{s}$ and $2 \mu \mathrm{s}$ symmetric waveforms had substantially higher lethal thresholds than the monopolar $100 \mu$ s waveform.

When the longest pulse in the waveform was either $1 \mu$ s or $2 \mu \mathrm{s}$, introducing asymmetry into the waveform by using a $0.5 \mu$ s pulse decreased the lethal threshold by an average of $25 \%$ and using a $0.25 \mu$ s pulse decreased the lethal threshold by an average of $45 \%$, compared to the associated symmetric (1-1-1 or 2-1-2) waveforms. Use of a monopolar waveform decreased the lethal threshold by an average of $56 \%$ compared to the associated symmetric waveforms. All asymmetric H-FIRE treatment groups which were monopolar or incorporated $0.25 \mu \mathrm{s}$ pulses had statistically significantly lower $(\alpha=0.01)$ lethal thresholds than the associated symmetric waveforms. Additionally, the lethal thresholds for U87 cells exposed to 1-1-0.5 and 2-1-0.5 waveforms were significantly lower than those exposed to 1-1-1 and 2-1-2 waveforms, respectively.

$50 \mu$ s versus $100 \mu s$ Energized Times. IRE is often employed clinically when tumors are in close proximity to critical nerves or blood vessels where tissue heating can result in deleterious outcomes. A simple way to minimize heating is to reduce the pulse-length (IRE) or energized time per burst (H-FIRE). Figure 5 shows the impact that reducing the energized time from $100 \mu \mathrm{s}$ to $50 \mu \mathrm{s}$ has on lethal thresholds for the U87 cell lines. The results for both cell lines are summarized in Table 1. The IRE waveforms in Fig. 5a which were energized for $100 \mu \mathrm{s}$ had lethal thresholds which were $20 \%$ lower on average than those energized for $50 \mu \mathrm{s}$.

For H-FIRE treatments (Table 1), most treatment groups which received bursts energized for $100 \mu \mathrm{s} \mathrm{had} \mathrm{statis-}$ tically significantly lower lethal thresholds than the $50 \mu$ s groups. However, this increase in energized time did not impact the lethal thresholds for U87 cells treated with 1-1-1 and 1-1-0.5 waveforms. Despite the relatively small differences found for these two waveforms, the lethal thresholds found for $100 \mu$ s H-FIRE treatments were $20 \%$ lower than those energized for $50 \mu$ s when averaged across all H-FIRE waveforms.

Reversible Electroporation Thresholds. The reversible electroporation thresholds observed immediately after treatment are presented in Table 2 for both cell types and are shown in Fig. 6 for U87 cells. For the 50 $\mu$ s monopolar, 25-1-25, 13-1-37, and 10-1-40 waveforms (Fig. 6a), the average reversible electroporation threshold of $303 \mathrm{~V} / \mathrm{cm}$ was $40 \%$ lower than the lethal threshold when averaged across both cell types. There was not a statistically significant difference between the reversible electroporation thresholds between these waveforms.

Interestingly, there was some variation in reversible electroporation thresholds with H-FIRE waveforms (Fig. 6b-d). The largest differences between reversible and lethal thresholds occurred for the 1-1-1 and 2-1-2 symmetric bipolar waveforms. The 2-1-2 waveform resulted in the largest difference of $837 \mathrm{~V} / \mathrm{cm}$ for U87 (49\% lower). In contrast, the $2 \mu \mathrm{s}$ monopolar waveform only had a difference of $289 \mathrm{~V} / \mathrm{cm}$ between the lethal and reversible electroporation thresholds and there was not a statistically significant difference for the 1-1-2 waveform for either cell type. 

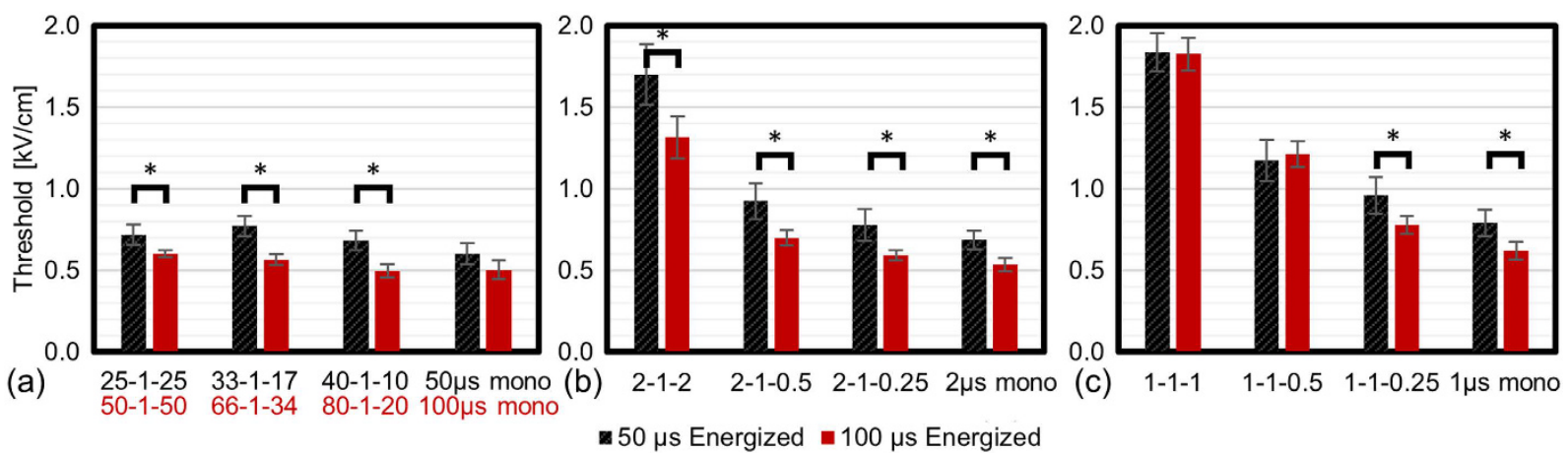

Figure 5. U87 lethal thresholds for treatments with $50 \mu$ s and $100 \mu$ s bursts. (a) Lethal threshold for long duration IRE pulses which were either monopolar $(50 \mu \mathrm{s}$ or $100 \mu \mathrm{s})$ or alternating polarity with a $1 \mu$ s delay between polarity change. Lethal thresholds for monopolar, symmetric, and asymmetric H-FIRE pulses where the longest pulse was (b) $2 \mu \mathrm{s}$ and (c) $1 \mu \mathrm{s}$. All treatment groups received 100x bursts where each burst was energized for $50 \mu \mathrm{s}$ (black) or $100 \mu \mathrm{s}$ (red). ${ }^{\star}$ Indicate groups which are statistically significantly different $(\alpha=0.01)$. Error bars represent one standard deviation from the mean. While doubling the energy delivered significantly decreased the lethal threshold for a majority treatment groups, waveform symmetry had a larger effect.

\begin{tabular}{|l|c|c|c|c|}
\hline \multirow{2}{*}{ Waveform } & \multicolumn{2}{|c|}{ U87 } & \multicolumn{2}{c|}{ MDA-MB-231 BR3 } \\
\cline { 2 - 5 } & Threshold [V/cm] & Lethal $\Delta$ & Threshold [V/cm] & Lethal $\Delta$ \\
\hline $25-1-25$ & $348 \pm 87$ & 370 & $569 \pm 84$ & 310 \\
\hline $33-1-17$ & $366 \pm 61$ & 407 & $606 \pm 100$ & 269 \\
\hline $40-1-10$ & $420 \pm 78$ & 264 & $605 \pm 32$ & 301 \\
\hline $50 \mu$ s Mono & $326 \pm 70$ & 277 & $554 \pm 9$ & 225 \\
\hline $2-1-2$ & $865 \pm 122$ & 837 & $864 \pm 62$ & 1164 \\
\hline $2-1-0.5$ & $587^{*} \pm 36$ & 338 & $679^{*} \pm 34$ & 621 \\
\hline $2-1-0.25$ & $548^{*} \pm 23$ & 232 & $593^{*} \pm 57$ & 378 \\
\hline $2 \mu$ s Mono & $400^{*} \pm 45$ & 289 & $476^{*} \pm 50$ & 528 \\
\hline $1-1-1$ & $848 \pm 137$ & 992 & $1188 \pm 171$ & 1573 \\
\hline $1-1-0.5$ & $679 \pm 94$ & 497 & $1330 \pm 314$ & 1452 \\
\hline $1-1-0.25$ & $645 \pm 53$ & 315 & $868 \pm 208$ & 395 \\
\hline $1 \mu$ s Mono & $422^{*} \pm 53$ & 371 & $579^{*} \pm 41$ & 472 \\
\hline $0.25-1-0.25$ & $2209^{\beta} \pm 243$ & -248 & $2021^{\delta} \pm 84$ & 967 \\
\hline $0.5-1-0.5$ & $1936^{\curlyvee} \pm 117$ & 218 & $1274 \pm 59$ & 1340 \\
\hline $0.5-1-0.25$ & $1263^{\beta \gamma} \pm 143$ & 55 & $1147^{\delta} \pm 226$ & 520 \\
\hline $1-1-2$ & $1294 \pm 202$ & -196 & $1102 \pm 313$ & 119 \\
\hline
\end{tabular}

Table 2. Reversible electroporation thresholds for U87 and MDA-MB-231 BR3 cell lines after exposure to 100x bursts of the listed waveforms. Each burst was energized for $50 \mu$ s and delivered at a repetition rate of $1 \mathrm{~Hz}$. Lethal $\Delta$ indicates the difference between the irreversible and reversible thresholds. Values are listed as mean \pm standard deviation. ${ }^{*}$ Indicates asymmetric waveforms which were found to be statistically significantly $(\alpha=0.01)$ different from their respective symmetric waveforms. ${ }^{\beta}, \gamma$, and ${ }^{\delta}$ symbols indicate sub-microsecond groups which were found to be statistically different from other sub-microsecond groups marked with the same symbol.

For $1 \mu \mathrm{s} \mathrm{H-FIRE} \mathrm{waveforms} \mathrm{(Fig.} \mathrm{6c),} \mathrm{the} \mathrm{1-1-1} \mathrm{waveform} \mathrm{resulted} \mathrm{in} \mathrm{the} \mathrm{largest} \mathrm{difference} \mathrm{of} 992 \mathrm{~V} / \mathrm{cm}(54 \%$ lower) for U87 cells between the reversible and lethal thresholds. The smallest difference between these two thresholds of $315 \mathrm{~V} / \mathrm{cm}$ was found for the 1-1-0.25 waveform.

Sub-microsecond H-FIRE protocols were also evaluated (Fig. 6d). For U87 cells there was no statistically significant difference between the reversible and lethal thresholds for the 0.25-1-0.25, 0.5-1-0.5, and 0.5-1-0.25 waveforms. However, the asymmetric 0.5-1-0.25 waveform had lower reversible and irreversible thresholds than the 0.25-1-0.25 and 0.5-1-0.5 symmetric waveforms.

U87 vs MDA-MB-231 BR3 Cell Lines. The lethal thresholds for U87 cells were lower than for MDA-MB-231 BR3 cells for all waveforms in Table 1, the mean thresholds were $36 \%$ lower for U87 cells. The largest difference observed (137\%) was for the 1-1-0.5 waveform when $100 \times 50 \mu$ s bursts were delivered. The smallest difference (13\%) was observed for the 33-1-17 waveform. The 50 and $100 \mu$ s IRE groups in Fig. 5a had 

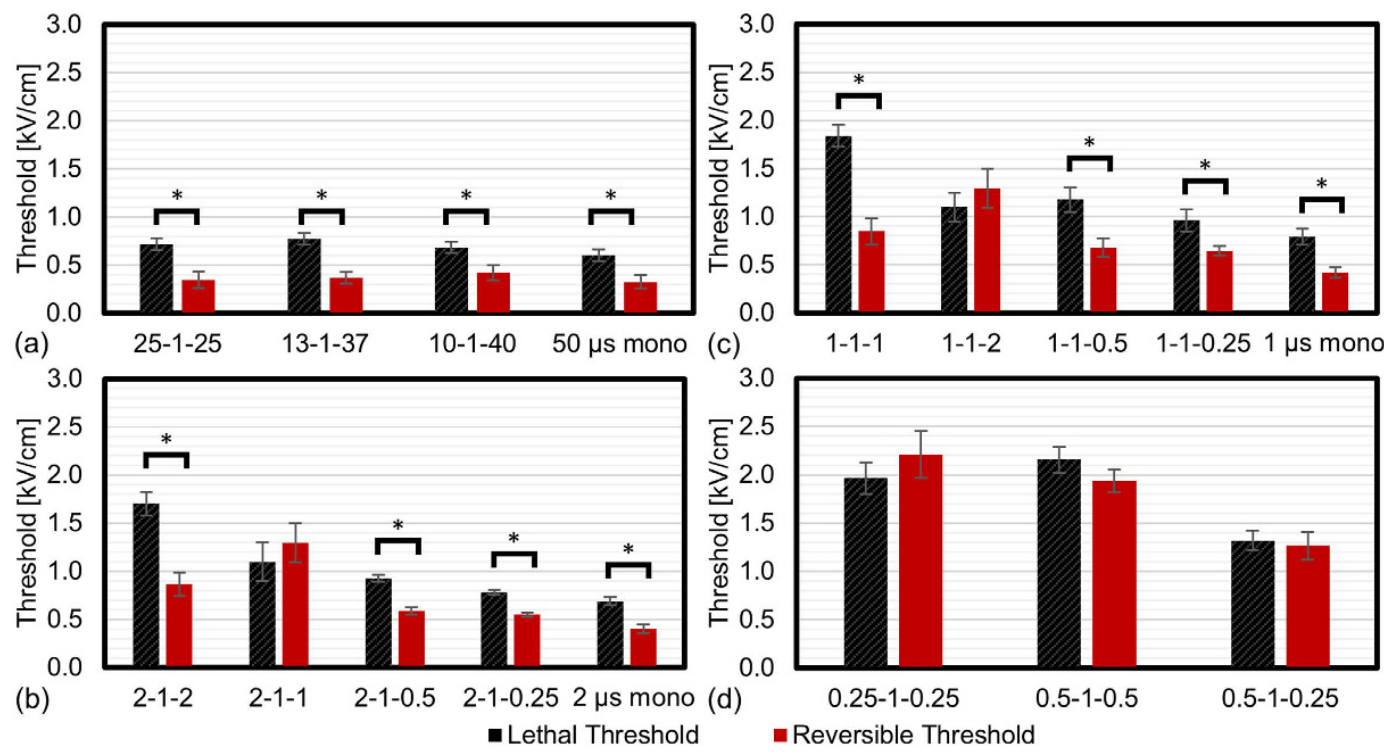

Figure 6. Pulse waveforms can be used to tune reversible and irreversible electroporation responses. Examination of reversible (red) and lethal (black) thresholds in 2D monolayer cultures of U87 cells for different $50 \mu$ s protocols. (a) Reversible and lethal thresholds for long duration $(>20 \mu \mathrm{s})$ IRE pulses which were either monopolar $(50 \mu \mathrm{s})$ or alternated with a $1 \mu \mathrm{s}$ delay between polarity change. Reversible and lethal thresholds for monopolar, symmetric, and asymmetric H-FIRE pulses where the longest pulse was (b) $2 \mu \mathrm{s}$, (c) $1 \mu \mathrm{s}$, and (d) $0.5 \mu \mathrm{s} .{ }^{*}$ Indicate groups which are statistically significantly different $(\alpha=0.01)$. Error bars represent one standard deviation from the mean. When the therapeutic intent is to induce reversible electroporation while minimizing cell death, 1-1-1 and 2-1-2 symmetric H-FIRE waveforms may be favorable due to the large difference between the reversible and irreversible electroporaiton thresholds for these waveforms.

the smallest average difference in lethal threshold between cell types at $26 \%$. The average difference between cell types increased to $31 \%$ for $2 \mu \mathrm{s}$ (Fig. 5b) and $49 \%$ for $1 \mu \mathrm{s}$ (Fig. 5c) H-FIRE waveforms.

The reversible electroporation thresholds were lower for U87 cells than for MDA-MB-231 BR3 cells in 11 of 16 waveforms evaluated in Table 2. Only the 0.5-1-0.5 waveform had a reversible electroporation threshold for U87 cells which was statistically significantly greater than for MDA-MB-231 BR3 cells. The 2-1-2, 1-1-2, 0.5-1-0.25, and 0.25-1-0.25 waveforms had also had mean reversible electroporation thresholds which were higher for the U87 cells, however these were not statistically significant different between cell types.

Modeling the Impact of Pulse Asymmetry on Clinical Ablations. Finite element models which incorporate the reversible and lethal electric field thresholds found here were used to predict how different H-FIRE waveforms may affect the size and geometry of ablations created using clinical ablation electrodes and pulse voltages used in the treatment of solid tumors. The methods for creating these models and validation against ablations created in ex vivo liver tissue are presented in the supplemental document. Symmetric H-FIRE waveforms required significantly higher electric fields to induce cell death compared to asymmetric waveforms. This resulted in the production of smaller predicted ablations for symmetric waveforms compared to the asymmetric waveforms when calculated using experimentally determined lethal thresholds (Supplemental Figure S1c-g).

A simulated treatment delivering $100 \times 3.0 \mathrm{kV} 50 \mu \mathrm{s}$ monopolar pulses through a $1 \mathrm{~cm}$ electrode exposure $(\mathrm{MP}$ Probe) predict an ablation measuring $1.7 \times 1.0 \mathrm{~cm}$. For the same $50 \mu \mathrm{s}$ energized time, the $2 \mu \mathrm{s} \mathrm{monopolar}$ waveform produced the largest simulated H-FIRE ablation of $1.6 \times 0.9 \mathrm{~cm}$ followed by $1.6 \times 0.8 \mathrm{~cm}, 1.5 \times 0.7 \mathrm{~cm}$, and $1.3 \times 0.4 \mathrm{~cm}$ for the 2-1-0.25, 2-1-0.5, and 2-1-2 waveforms, respectively (Supplemental Table S1). Volumetrically, ablations created by the $2 \mu$ s monopolar waveforms were $6.3 x$ larger than the symmetric 2-1-2 waveform.

Increasing the energized time from $50 \mu$ s to $100 \mu$ s resulted in a decrease in lethal threshold of approximately $20 \%$ averaged across all waveforms evaluated in this study. $1.8 \times 1.1 \mathrm{~cm}, 1.7 \times 1.0 \mathrm{~cm}, 1.7 \times 1.0 \mathrm{~cm}, 1.6 \times 0.9 \mathrm{~cm}$, $1.4 \times 0.5 \mathrm{~cm}$ simulated ablation zones were created for the $100 \mu \mathrm{s}$ mono $(505 \mathrm{~V} / \mathrm{cm}), 2 \mu \mathrm{s}$ mono $(536 \mathrm{~V} / \mathrm{cm}), 2-1-$ $0.25(594 \mathrm{~V} / \mathrm{cm}), 2-1-0.5(700 \mathrm{~V} / \mathrm{cm})$, and 2-1-2 $(1316 \mathrm{~V} / \mathrm{cm})$ waveforms, respectively. Under these conditions, the $2 \mu \mathrm{s}$ monopolar waveform produced a simulated ablation volume which was $5.8 \mathrm{x}$ larger than the symmetric 2-1-2 waveform.

Ex Vivo Results versus Numerical Predictions. Experimental ablations were created in ex vivo porcine liver by delivering $100 \times 3.5 \mathrm{kV}$ bursts of the 2-1-2 waveform (Supplemental Figure S2). Each burst was energized for $100 \mu \mathrm{s}$ and bursts were delivered at a repetition frequency of $1 \mathrm{~Hz}$. Experimental ablations created using the clinical MP probe with a $1 \mathrm{~cm}$ electrode exposure had an average length of $1.3 \pm 0.3 \mathrm{~cm}$ and an average width of $0.7 \pm 0.2 \mathrm{~cm}$ (Supplemental Figure S3). The numerical model of the MP probe predicted an ablation measuring $1.4 \times 0.6 \mathrm{~cm}$ when these experimental conditions were simulated (Supplemental Table S2) and the results were within $0.1 \mathrm{~cm}$ of the average ablation created experimentally $(1.3 \times 0.7 \mathrm{~cm})$. Experimental ablations 
created using the clinical BP probe had an average length of $1.4 \pm 0.1 \mathrm{~cm}$ and an average width of $0.9 \pm 0.2 \mathrm{~cm}$ (Supplemental Figure S3). The numerical model of the BP probe predicted an ablation measuring $1.2 \times 0.7 \mathrm{~cm}$ using these experimental parameters (Supplemental Table S3) and the results were within $0.2 \mathrm{~cm}$ of the average ablation created experimentally $(1.4 \times 0.9 \mathrm{~cm})$. This difference of one to two millimeters in length and width between the average experimental and predicted ablations serve as a preliminary validation of the ability of these models, which incorporate dynamic tissue conductivity with reversible and lethal thresholds found in vitro, to predict ablation dimensions in tissue.

\section{Discussion}

2D versus 3D Culture Conditions. H-FIRE is a relatively new electroporation protocol. The symmetric waveforms were first proposed by Arena et al. as a method for creating more uniform ablations in heterogeneous tissues $^{23}$. Early in vitro experiments on cells in suspension showed that these waveforms required substantially higher electric fields $(2-2.7 \mathrm{x})$ to induce cell death versus the standard $100 \mu \mathrm{s}$ monopolar IRE waveforms ${ }^{29}$. When cells were grown in 3D collagen tumor models the lethal electric field threshold decreased to $1.5 \mathrm{x}$ and $2.2 \mathrm{x}$ the lethal threshold for equivalent energy $2 \mu \mathrm{s}$ and $1 \mu$ s symmetric H-FIRE protocols, respectively. It is hypothesized that the decrease in the ratios of lethal thresholds between IRE and H-FIRE protocols is likely due to cells transforming from a spherical shape in suspension into a more complex natural geometry when cultured in 3D models ${ }^{29}$.

Arena $e$ al. found a lethal threshold of $500 \mathrm{~V} / \mathrm{cm}$ for pancreatic cancer cells when delivering $80 \times 100 \mu \mathrm{s}$ monopolar IRE pulses in this 3D model ${ }^{30}$. The lethal thresholds for U87 brain cancer cells found in this study were $463 \mathrm{~V} / \mathrm{cm}$ and $505 \mathrm{~V} / \mathrm{cm}$ in $3 \mathrm{D}$ and $2 \mathrm{D}$ models, respectively, when $100 \times 100 \mu$ s monopolar IRE pulses were delivered. Previous studies found lethal thresholds in liver tissue between 300 and $640 \mathrm{~V} / \mathrm{cm}$ using similar IRE protocols ${ }^{36-38}$, indicating that both the $2 \mathrm{D}$ and $3 \mathrm{D}$ culture models recapitulate effects seen in mammalian tissue. Fourteen of sixteen protocols conducted in 2D and 3D conditions showed no statistically significant difference in lethal threshold indicating that the 3D model has a limited effect on the lethal threshold and cells cultured in a 2D monolayer are a relatively effective model for studying H-FIRE. 2D monolayers are a convenient model as they are relatively simple to generate, are less expensive, and less time intensive to create compared to 3D cultures. However, when the thresholds were statistically different between groups, the 3D cultures all had lower lethal thresholds. This indicates that further investigation in 3D constructs may be warranted, especially if in vivo results are found to differ significantly from those found in 2D monolayers.

U87 vs MDA-MB-231 BR3 Cell Lines. The MDA-MB-231 BR3 cells appeared to stretch out into a flattened oval shape with few protrusions. These cells continued to divide as the cultures approached confluency and appeared to overlap with each other to cover the entire well plate surface. In contrast, the U87 cells formed more star shaped geometries with multiple elongated protrusions. They formed a web of interconnected cells and cell division slowed as they became confluent. The networks of U87 cells had numerous gaps of unoccupied well plate area. While not extensively examined here, the differences in lethal thresholds observed between cell types may be due to differences in cell geometry affecting the charging time of the cell membrane. Ivey et al. found identical lethal thresholds for U87, NHA, and D1TNC1 cell lines after treatment with $100 \mu$ s monopolar pulses, but significantly different lethal thresholds between U87 cells and the astrocyte (NHA and D1TNC1) cell lines when using a 1-5-1 H-FIRE waveform ${ }^{26}$. These differences in lethal threshold were hypothesized to be a function of the morphology of the cell including the nucleus-to-cytoplasm ratio ${ }^{26}$ and morphological factors may have impacted the observed differences between U87 and MDA-MB-231 BR3 cells shown here.

Effects of Waveform Asymmetry and Energized Time. For H-FIRE, waveform asymmetry appeared to be the biggest factor affecting the lethal threshold. Doubling the dose delivered by energizing the bursts for $100 \mu \mathrm{s}$ rather than $50 \mu \mathrm{s}$ decreased the lethal threshold by an average of $20 \%$. In contrast, the lethal thresholds for asymmetric waveforms were $42 \%$ lower than the symmetric waveforms, averaged across all H-FIRE groups. Waveforms incorporating a $0.5 \mu$ s or $0.25 \mu$ s pulses were $25 \%$ and $45 \%$ lower than symmetric waveforms, respectively. When only monopolar $1 \mu \mathrm{s}$ or $2 \mu \mathrm{s}$ pulses were used in the burst, the lethal thresholds were $56 \%$ lower on average compared to their respective symmetric (1-1-1 or 2-1-2) counterparts.

We hypothesize that two potential mechanisms contribute to the decrease in lethal threshold when asymmetric waveforms are used: a cumulative charging of the cell membrane and enhanced electrokinetic transport. Symmetric pulses may be inducing a cancellation effect ${ }^{39}$ in which the alternating polarity pulses sequentially charge then discharge the potential across the cell membrane faster than this potential would dissipate in the absence of an alternating pulse. The symmetric H-FIRE waveforms had the largest differences between reversible and lethal thresholds indicating that these waveforms are effective at facilitating pore formation, but the rapid charging and discharging of the membrane may not have efficiently driven the pore expansion processes necessary to permanently disrupt the cell membrane. When asymmetric waveforms are used, the forced discharge caused by the alternate polarity pulse occurs to a lesser extent than for symmetric waveforms resulting in a cumulative charging of the cell membrane by the longer duration pulses in the burst. This cumulative charging likely increases the potential across the cell membrane above the threshold required for pore formation and then then drives pore expansion.

Monopolar IRE pulses act as a baseline to indicate the lowest electroporation thresholds possible under these experimental conditions. Monopolar H-FIRE waveforms which contained $5 \mu$ s or $2 \mu$ s pulses had nearly identical lethal thresholds to equivalent 50 or $100 \mu$ s pulses. This indicates that the induced membrane potential does not decrease substantially during the $1 \mu$ s delay between subsequent pulses in a monopolar H-FIRE burst. However, $1 \mu$ s monopolar H-FIRE waveforms were less efficient at inducing cell death than $2 \mu$ s or $5 \mu$ s monopolar waveforms indicating that this may be the lower limit for inducing pore expansion. 
This $1 \mu$ s threshold is likely related to the charging time of the cell membrane which is dependent on a number of factors including cell geometry and medium conductivity, but is typically calculated to be approximately $1 \mu s^{29,40}$. Previous studies on sub-microsecond pulses showed that increasing the number of monopolar pulses increased the number, but not the size of nanopores formed in the membrane ${ }^{41}$. A similar phenomena may be occurring here in which continued delivery of pulses $1 \mu$ s or shorter leads to the formation of numerous smaller pores rather than rapid cell membrane destruction caused by pore expansion and any lethal effects are due to mechanisms which occur post-treatment.

Symmetric pulses may also be inducing a cancelation effect which results in zero net movement of charged species. The introduction of asymmetry in the H-FIRE waveform may result in net electrokinetic transport of charged molecules across the cell membrane resulting in conditions which make it challenging for the cell to regain homeostasis. It was found that monopolar $300 \mathrm{~ns}$ pulses resulted in a higher degree of calcium mobilization and lower viabilities 24 hours post treatment compared to bipolar waveforms with inter-pulse delays of up to $10 \mu \mathrm{s}^{39}$. Additionally, $100 \mu \mathrm{s}$ monopolar pulses resulted in significantly higher magnitudes of PI influx compared to equivalent 1-1-1 H-FIRE waveforms ${ }^{42}$ for cells in suspension. A combination of these phenomena may be occurring with asymmetric $\mathrm{H}$-FIRE waveforms resulting in the formation of larger pores due to pore expansion and increased molecular transport due to electrophoresis, however, the degree to which these phenomena occur have yet to be investigated for asymmetric H-FIRE waveforms.

Reversible Electroporation. Typical ECT ${ }^{32}$ and EGT ${ }^{43,44}$ protocols use 2 to $24^{45}$ monopolar pulses between $100 \mu \mathrm{s}^{46}$ and $20 \mathrm{~ms}^{47}$ to electroporate cells and electrophoretically drive molecules across the cell membrane. A challenge with these monopolar waveforms is that they induce some degree of irreversible electroporation in vivo due to the relatively small difference between their reversible and lethal thresholds. Previous studies by Miklavcic et. al found a difference of $275 \mathrm{~V} / \mathrm{cm}$ between the reversible and lethal thresholds in in vivo rabbit liver tissue after the delivery of eight $10 \mu$ s pulses ${ }^{27}$ and the monopolar IRE pulses investigated in this study had approximately $225-277 \mathrm{~V} / \mathrm{cm}$ between the reversible and lethal thresholds. From a clinical prospective, symmetric H-FIRE waveforms may be more advantageous in ECT and EGT protocols, where the intent is to transfer membrane impermeable material into the cell rather than inducing cell death, because the symmetric waveforms have a very large difference between their reversible and irreversible thresholds $(837-1573 \mathrm{~V} / \mathrm{cm})$.

It is unclear why symmetric waveforms resulted in a larger difference between reversible and irreversible thresholds. However, we hypothesize that the rapid charging and discharging of the cell membrane by the symmetric pulses inhibits pore expansion and instead results in an increase in the number, but not size of the pores formed ${ }^{41}$. However, it is unclear if these symmetric waveforms will be capable of transporting molecules across biological membranes efficiently. Numerical simulations indicate that electrophoretic and diffusive transport of molecules play roughly equivalent roles in the transport of small molecules following electroporation ${ }^{48}$ while electrophoresis ${ }^{49}$ and electroporation induced endocytosis ${ }^{50}$ are implicated in the transport of larger molecules ${ }^{51}$.

Symmetric bipolar wave forms were recently reported to be an effective means for transiently disrupting the blood brain barrier ${ }^{52}$, however, reversible electroporation for chemotherapy and gene transfer have yet to be demonstrated in vitro or in vivo with these waveforms. We hypothesize that the introduction of a small degree of asymmetry into the H-FIRE waveform (e.g. a 2.0-1-2.1 waveform) may be sufficient to induce electrophoretic movement of charged molecules without producing large lethal ablation zones or inducing muscle contractions ${ }^{53}$. Andre et al. demonstrated that high voltage pulses to induce electroporation followed by low voltage pulses to enhance electrophoretic transport were advantageous for in vivo $\mathrm{EGT}^{49}$ and a similar strategy may be necessary with H-FIRE. However, further work will be necessary to determine optimal protocols for using H-FIRE waveforms for these non-lethal protocols.

Modeling the Impact of Pulse Asymmetry on Clinical Ablations. The use of numerical models for predictive treatment planning is relatively common for IRE procedures ${ }^{8,11,17,54,55}$, in part because real time ultrasound visualization of the ablation zones is challenging and follow up CT or MRI imaging may be required to confirm total tumor coverage ${ }^{56}$. The model presented here builds upon previous dynamic conductivity models ${ }^{11,17,34}$ by incorporating reversible and lethal thresholds found in vitro into the dynamic conductivity function. Given recent data which indicates that H-FIRE may be able to preferentially target malignant cells ${ }^{26}$, this modeling approach may be necessary for predicting ablations margins created in in vivo.

Finite element models simulating clinical electrodes and treatment voltages (Supplemental Figure S1) indicate that asymmetric H-FIRE waveforms can potentially be used to produce equivalent ablation volumes to standard IRE waveforms (Supplemental Table S1). A $3 \mathrm{kV}$ treatment using a 2-1-2 waveform energized for $100 \mu$ s produced a $1.4 \times 0.5 \mathrm{~cm}$ simulated ablation $\left(0.18 \mathrm{~cm}^{3}\right.$ volume $)$ as shown in Supplemental Figure S1g. In contrast, switching to a 2-1-0.25 waveform would increase the ablation volume 5.5 -fold to $1.0 \mathrm{~cm}^{3}(1.7 \times 1.1 \mathrm{~cm})$.

Clinically, a single electrode and grounding pad configuration is not implemented because $100 \mu s$ pulses would stimulate muscles over such a wide of a volume of the body that chemical paralytics may no longer be sufficient to eliminate muscle contractions. Symmetric H-FIRE waveforms have been shown to mitigate these contractions $^{24}$ and eliminate the need for chemical paralytics in large animal studies ${ }^{25}$ at the expense of creating smaller ablations ${ }^{25}$. Extensive in vivo investigation will be necessary to evaluate if muscle contractions are present when asymmetric H-FIRE waveforms are used or if chemical paralytics will be required to mitigate them. However, based on previous studies it is feasible that asymmetric H-FIRE waveforms will be able to produce clinically relevant ablations using a single electrode.

Study Limitations. There are a number of important limitations to the current study. $2 \mathrm{D}$ and $3 \mathrm{D}$ in vitro cell cultures have been found to adequately recapitulate the lethal electric field thresholds required for IRE treatments to induce cell death in vivo. However, data on in vivo lethal thresholds for H-FIRE treatments have yet to 
be published and it is unknown if values determined in vitro will match those found in vivo. Here and in prior studies $^{25,30}$, lethal thresholds for IRE and H-FIRE waveforms were investigated approximately 24 hours post treatment. The mechanisms of cell death following H-FIRE are unclear, but appear to be a combination of physical necrosis due to cell membrane destruction and apoptosis. However, mechanistic studies conducted for durations longer than 24 hours have yet to be reported. Preliminary studies on cells in suspension showed that viability after H-FIRE treatment continued to decline between 1 and 24 hours post treatment ${ }^{29}$ and it is unclear if the ablation zones created in $2 \mathrm{D}$ or $3 \mathrm{D}$ culture would continue to evolve over longer time periods. Theoretical clinical ablation zones were generated using numerical models which have previously been used for clinical treatment planning ${ }^{17}$ combined with experimental electroporation threshold data generated in $2 \mathrm{D}$ culture. These models predict that asymmetric waveforms may be useful in increasing the size of H-FIRE ablations in vivo, however, extensive experimental examination will be required to validate these results.

\section{Conclusion}

In vitro models of primary and metastatic brain cancer were used to show that the lethal threshold for H-FIRE treatments is affected by the symmetry of the waveform. Asymmetric waveforms, including monopolar waveforms, have significantly lower lethal thresholds than equivalent energy symmetric waveforms. The use of asymmetric H-FIRE waveforms clinically should result in the creation of equivalent ablation volumes to those seen in IRE procedures while mitigating muscle contractions caused by long duration pulses. These results indicate that in vivo testing to determine anti-tumor efficacy of asymmetric H-FIRE waveforms is warranted.

\section{References}

1. Davalos, R. V., Mir, L. M. \& Rubinsky, B. Tissue ablation with irreversible electroporation. Annals of Biomedical Engineering 33, 223-231 (2005).

2. Scheffer, H. J. et al. Irreversible Electroporation for Nonthermal Tumor Ablation in the Clinical Setting: A Systematic Review of Safety and Efficacy. J Vasc Interv Radiol 25, 997-1011 (2014).

3. Szot, C. S., Buchanan, C. F., Freeman, J. W. \& Rylander, M. N. 3D in vitro bioengineered tumors based on collagen I hydrogels. Biomaterials 32, 7905-7912 (2011).

4. Dunki-Jacobs, E., Philips, P. \& Martin, I. Evaluation of thermal injury to liver, pancreas and kidney during irreversible electroporation in an in vivo experimental model. Br J Surg 101, 1113-1121 (2014).

5. Martin, R. C., McFarland, K., Ellis, S. \& Velanovich, V. Irreversible electroporation therapy in the management of locally advanced pancreatic adenocarcinoma. J Am Coll Surgeons 215, 361-369 (2012).

6. Martin, R. C., McFarland, K., Ellis, S. \& Velanovich, V. Irreversible electroporation in locally advanced pancreatic cancer: potential improved overall survival. Ann Surg Oncol 20, 443-449 (2013).

7. Martin, R. C. et al. Treatment of 200 locally advanced (stage III) pancreatic adenocarcinoma patients with irreversible electroporation: safety and efficacy. Ann Surg 262, 486-494 (2015).

8. Neal, R. E. et al. In Vivo Irreversible Electroporation Kidney Ablation: Experimentally Correlated Numerical Models. Biomedical Engineering, IEEE Transactions on 62, 561-569 (2015).

9. Onik, G., Mikus, P. \& Rubinsky, B. Irreversible electroporation: implications for prostate ablation. Technol Cancer Res Treat 6, 295-300 (2007).

10. Rubinsky, J., Onik, G., Mikus, P. \& Rubinsky, B. Optimal Parameters for the Destruction of Prostate Cancer Using Irreversible Electroporation. The Journal of Urology 180, 2668-2674 (2008).

11. Neal, R. E. et al. In vivo characterization and numerical simulation of prostate properties for non-thermal irreversible electroporation ablation. The Prostate 74, 458-468 (2014).

12. Valerio, M. et al. Initial assessment of safety and clinical feasibility of irreversible electroporation in the focal treatment of prostate cancer. Prostate Cancer Prostatic Dis 17, 343-347 (2014).

13. Tomihama, R., Günther, E., Kim, D. \& Stehling, M. Irreversible electroporation treatment for prostate adenocarcinomas: a safety outcome study. Journal of Vascular and Interventional Radiology 26, S121-S122 (2015).

14. Garcia, P. A. et al. Intracranial nonthermal irreversible electroporation: in vivo analysis. J Membr Biol 236, 127-136 (2010).

15. Ellis, T. L. et al. Nonthermal irreversible electroporation for intracranial surgical applications: laboratory investigation. Journal of neurosurgery 114, 681-688 (2011)

16. Garcia, P. A., Rossmeisl, J. H., Ellis, T. L. \& Davalos, R. V. Nonthermal Irreversible Electroporation as a Focal Ablation Treatment for Brain Cancer. In Tumors of the Central Nervous System. Volume 12 171-182 (Springer, 2014).

17. Rossmeisl, J. H. Jr et al. Safety and feasibility of the NanoKnife system for irreversible electroporation ablative treatment of canine spontaneous intracranial gliomas. Journal of neurosurgery 123, 1008-1025 (2015).

18. Thomson, K. R. et al. Investigation of the safety of irreversible electroporation in humans. J Vasc Interv Radiol 22, 611-621 (2011).

19. Martin, R. C., Schwartz, E., Adams, J., Farah, I. \& Derhake, B. M. Intra - operative Anesthesia Management in Patients Undergoing Surgical Irreversible Electroporation of the Pancreas, Liver, Kidney, and Retroperitoneal Tumors. Anesth Pain Med 5, e22786 (2015).

20. Golberg, A., Bruinsma, B. G., Uygun, B. E. \& Yarmush, M. L. Tissue heterogeneity in structure and conductivity contribute to cell survival during irreversible electroporation ablation by electric field sinks. Scientific reports $\mathbf{5}$ (2015).

21. Gabriel, C. Compilation of the Dielectric Properties of Body Tissues at RF and Microwave Frequencies. (DTIC Document, 1996).

22. Bhonsle, S. P., Arena, C. B., Sweeney, D. C. \& Davalos, R. V. Mitigation of Impedance Changes Due to Electroporation Therapy Using Bursts of High-Frequency Bipolar Pulses. Biomed Eng Online doi: 10.1186/1475-925X-14-S3-S3 (2015).

23. Arena, C. B., Sano, M. B., Rylander, M. N. \& Davalos, R. V. Theoretical considerations of tissue electroporation with high-frequency bipolar pulses. IEEE Trans Biomed Eng 58, 1474-1482 (2011).

24. Arena, C. B. et al. High-frequency irreversible electroporation (H-FIRE) for non-thermal ablation without muscle contraction. Biomed Eng Online 10, 102 (2011).

25. Sano, M. B. et al. Bursts of Bipolar Microsecond Pulses Inhibit Tumor Growth. Scientific reports 5 (2015).

26. Ivey, J. W. et al. Targeted cellular ablation based on the morphology of malignant cells. Scientific reports 5 (2015).

27. Miklavcic, D., Semrov, D., Mekid, H. \& Mir, L. M. A validated model of in vivo electric field distribution in tissues for electrochemotherapy and for DNA electrotransfer for gene therapy. Biochim Biophys Acta 1523, 73-83 (2000).

28. Edd, J. F., Horowitz, L., Davalos, R. V., Mir, L. M. \& Rubinsky, B. In vivo results of a new focal tissue ablation technique: irreversible electroporation. IEEE Trans Biomed Eng 53, 1409-1415 (2006).

29. Sano, M. B., Arena, C. B., DeWitt, M. R., Saur, D. \& Davalos, R. V. In-vitro bipolar nano- and microsecond electro-pulse bursts for irreversible electroporation therapies. Bioelectrochemistry 100, 69-79 (2014).

30. Arena, C. B., Szot, C. S., Garcia, P. A., Rylander, M. N. \& Davalos, R. V. A three-dimensional in vitro tumor platform for modeling therapeutic irreversible electroporation. Biophys J 103, 2033-2042 (2012). 
31. Miklavcic, D. et al. Electrochemotherapy: technological advancements for efficient electroporation-based treatment of internal tumors. Medical \& Biological Engineering \& Computing 50, 1213-1225 (2012).

32. Sersa, G. et al. Electrochemotherapy of tumors as in situ vaccination boosted by immunogene electrotransfer. Cancer Immunol Immunother 64, 1315-1327 (2015).

33. Kim, L. S., Huang, S., Lu, W., Lev, D. C. \& Price, J. E. Vascular endothelial growth factor expression promotes the growth of breast cancer brain metastases in nude mice. Clin Exp Metastasis 21, 107-118 (2004).

34. Sel, D. et al. Sequential finite element model of tissue electropermeabilization. IEEE Transactions on Biomedical Engineering 52, 816-827 (2005)

35. Sano, M. B., Fan, R. E., Hwang, G. L., Sonn, G. A. \& Xing, L. Production of Spherical Ablations Using Nonthermal Irreversible Electroporation: A Laboratory Investigation Using a Single Electrode and Grounding Pad. J Vasc Interv Radiol 27, 1432-1440 e1433 (2016).

36. Sano, M. B. et al. Towards the creation of decellularized organ constructs using irreversible electroporation and active mechanical perfusion. Biomedical Engineering Online 9, 83 (2010).

37. Edd, J. F., Horowitz, L., Davalos, R. V., Mir, L. M. \& Rubinsky, B. In vivo results of a new focal tissue ablation technique: Irreversible electroporation. Ieee T Bio-Med Eng 53, 1409-1415 (2006).

38. Miklavčič, D., Šemrov, D., Mekid, H. \& Mir, L. M. A validated model of in vivo electric field distribution in tissues for electrochemotherapy and for DNA electrotransfer for gene therapy. Biochimica et Biophysica Acta (BBA)-General Subjects 1523, 73-83 (2000).

39. Pakhomov, A. G. et al. Cancellation of cellular responses to nanoelectroporation by reversing the stimulus polarity. Cell Mol Life Sci 71, 4431-4441 (2014).

40. Kotnik, T. \& Miklavčič, D. Theoretical evaluation of voltage inducement on internal membranes of biological cells exposed to electric fields. Biophysical Journal 90, 480-491 (2006).

41. Pakhomov, A. G. et al. Multiple nanosecond electric pulses increase the number but not the size of long-lived nanopores in the cell membrane. Biochimica et Biophysica Acta (BBA)-Biomembranes 1848, 958-966 (2015).

42. Sweeney, D. C. et al. Quantification of cell membrane permeability induced by monopolar and high frequency bipolar bursts of electrical pulses. Biochimica et Biophysica Acta (BBA)-Biomembranes (2016).

43. Forde, P. F. et al. Non-viral immune electrogene therapy induces potent antitumour responses and has a curative effect in murine colon adenocarcinoma and melanoma cancer models. Gene Ther 22, 29-39 (2015).

44. Kaneko, T., Sakuma, T., Yamamoto, T. \& Mashimo, T. Simple knockout by electroporation of engineered endonucleases into intact rat embryos. Scientific reports 4, 6382 (2014).

45. Kos, S. et al. Improved specificity of gene electrotransfer to skin using pDNA under the control of collagen tissue-specific promoter. The Journal of membrane biology 248, 919-928 (2015).

46. Mir, L. M. et al. Standard operating procedures of the electrochemotherapy: instructions for the use of bleomycin or cisplatin administered either systemically or locally and electric pulses delivered by the Cliniporator TM by means of invasive or non-invasive electrodes. Eur J Cancer Suppl 4, 14-25 (2006).

47. Cutrera, J., Dibra, D., Xia, X. \& Li, S. Enhancement of reporter gene detection sensitivity by insertion of specific mini-peptidecoding sequences. Cancer Gene Ther 17, 131-140 (2010).

48. Schoenbach, K. H. et al. Ion transport into cells exposed to monopolar and bipolar nanosecond pulses. Bioelectrochemistry 103, 44-51 (2015)

49. Andre, F. et al. Efficiency of high-and low-voltage pulse combinations for gene electrotransfer in muscle, liver, tumor, and skin. Hum Gene Ther 19, 1261-1272 (2008).

50. Glogauer, M., Lee, W. \& McCulloch, C. Induced endocytosis in human fibroblasts by electrical fields. Exp Cell Res 208, 232-240 (1993).

51. Escoffre, J.-M. et al. What is (still not) known of the mechanism by which electroporation mediates gene transfer and expression in cells and tissues. Mol Biotechnol 41, 286-295 (2009).

52. Arena, C. B. et al. Focal blood-brain-barrier disruption with high-frequency pulsed electric fields. Technology 2, 206-213 (2014).

53. Miklavčič, D. et al. The effect of high frequency electric pulses on muscle contractions and antitumor efficiency in vivo for a potential use in clinical electrochemotherapy. Bioelectrochemistry 65, 121-128 (2005).

54. Neal, R. E. et al. Treatment of breast cancer through the application of irreversible electroporation using a novel minimally invasive single needle electrode. Breast Cancer Res Treat 123, 295-301 (2010).

55. Neal, R. E., Garcia, P., Robertson, J. L. \& Davalos, R. V. Experimental characterization and numerical modeling of tissue electrical conductivity during pulsed electric fields for irreversible electroporation treatment planning. Biomedical Engineering, IEEE Transactions on 59, 1076-1085 (2012).

56. Neal Ii, R. E., Cheung, W., Kavnoudias, H. \& Thomson, K. R. Spectrum of imaging and characteristics for liver tumors treated with irreversible electroporation. Journal of Biomedical Science and Engineering 05, 813-818 (2012).

\section{Acknowledgements}

This work was supported in part by the DOD through the Prostate Cancer Research Program (PCRP) Postdoctoral Training Award W81XWH-15-1-0137

\section{Author Contributions}

M.S. conceived of, designed, developed, and conducted the experiments. M.S., R.F. and L.X. analyzed and interpreted the data. L.X. supervised the study. All authors contributed to writing, editing, and review of the manuscript.

\section{Additional Information}

Supplementary information accompanies this paper at http://www.nature.com/srep

Competing financial interests: MBS and LX have pending patents related to H-FIRE technologies. The other authors disclose no potential conflicts of interest.

How to cite this article: Sano, M. B. et al. Asymmetric Waveforms Decrease Lethal Thresholds in High Frequency Irreversible Electroporation Therapies. Sci. Rep. 7, 40747; doi: 10.1038/srep40747 (2017).

Publisher's note: Springer Nature remains neutral with regard to jurisdictional claims in published maps and institutional affiliations. 
(c) (i) This work is licensed under a Creative Commons Attribution 4.0 International License. The images or other third party material in this article are included in the article's Creative Commons license, unless indicated otherwise in the credit line; if the material is not included under the Creative Commons license, users will need to obtain permission from the license holder to reproduce the material. To view a copy of this license, visit http://creativecommons.org/licenses/by/4.0/

(C) The Author(s) 2017 